\title{
Toxicon
}

Toxicon 51 (2008) 1197-1206

www.elsevier.com/locate/toxicon

\section{Tx2-6 toxin of the Phoneutria nigriventer spider potentiates rat erectile function is}

\author{
K.P. Nunes ${ }^{\mathrm{a}}$, A. Costa-Gonçalves ${ }^{\mathrm{a}}$, L.F. Lanza ${ }^{\mathrm{a}}$, S.F. Cortes ${ }^{\mathrm{b}}$, M.N. Cordeiro ${ }^{\mathrm{c}}$, \\ M. Richardson ${ }^{c}$, A.M.C. Pimenta ${ }^{d}$, R.C. Webb ${ }^{\mathrm{e}}$, R. Leite ${ }^{\mathrm{e}}$, M.E. De Lima ${ }^{\mathrm{d}}{ }^{*}$ \\ ${ }^{a}$ Departamento de Fisiologia e Biofisica, Instituto de Ciências Biológicas, Universidade Federal de Minas Gerais , \\ Belo Horizonte, Minas Gerais, Brazil \\ ${ }^{\mathrm{b}}$ Departamento de Farmacologia, Instituto de Ciências Biológicas, Universidade Federal de Minas Gerais, \\ Belo Horizonte, Minas Gerais, Brazil \\ ${ }^{\mathrm{c}}$ FUNED, Fundação Ezequiel Dias, Belo Horizonte, MG, Brazil \\ ${ }^{\mathrm{d}}$ Departamento de Bioquímica e Imunologia, Instituto de Ciências Biológicas, Laboratório de Venenos e Toxinas Animais, \\ Universidade Federal de Minas Gerais, Av. Antonio Carlos, 6627, 31.270-901 Belo Horizonte, Minas Gerais, Brazil \\ ${ }^{\mathrm{e}}$ Department of Physiology, School of Medicine, Medical College of Georgia, GA, USA
}

Received 26 October 2007; received in revised form 7 February 2008; accepted 8 February 2008

Available online 26 February 2008

\begin{abstract}
The venom of the spider Phoneutria nigriventer contains several toxins that have bioactivity in mammals and insects. Accidents involving humans are characterized by various symptoms including penile erection. Here we investigated the action of Tx2-6, a toxin purified from the $P$. nigriventer spider venom that causes priapism in rats and mice. Erectile function was evaluated through changes in intracavernosal pressure/mean arterial pressure ratio (ICP/MAP) during electrical stimulation of the major pelvic ganglion (MPG) of normotensive and deoxycorticosterone-acetate (DOCA)-salt hypertensive rats. Nitric oxide (NO) release was detected in cavernosum slices with fluorescent dye (DAF-FM) and confocal microscopy. The effect of Tx2-6 was also characterized after intracavernosal injection of a non-selective nitric oxide synthase (NOS) inhibitor, L-NAME. Subcutaneous or intravenous injection of Tx2-6 potentiated the elevation of ICP/MAP induced by ganglionic stimulation. L-NAME inhibited penile erection and treatment with Tx2-6 was unable to reverse this inhibition. Tx2-6 treatment induced a significant increase of NO release in cavernosum tissue. Attenuated erectile function of DOCA-salt hypertensive rats was fully restored after toxin injection. Tx2-6 enhanced erectile function in normotensive and DOCA-salt hypertensive rats, via the NO pathway. Our studies suggest that Tx2-6 could be important for development of new pharmacological agents for treatment of erectile dysfunction.
\end{abstract}

(C) 2008 Elsevier Ltd. All rights reserved.

Keywords: Phoneutria nigriventer; Tx2-6 toxin; Nitric oxide; Erectile function

Ethical Statement: All experimental protocols were approved by the Ethics Committee of the Federal University of Minas Gerais (Process no. CETEA 22/2006) and followed the guidelines of the International Society of Toxinology, concerning the use of experimental animals, in this case rats (IST, Toxicon 30:1-2, 1992). No pain was inflicted to the animals.

*Corresponding author. Tel.: + 553134092659 ; fax: + 553134092614.

E-mail address: delima@icb.ufmg.br (M.E. De Lima). 


\section{Introduction}

Penile erection is a complex response that occurs under regulatory control. It is initiated by activation of parasympathetic pelvic nerves leading to arterial dilatation followed by relaxation of the corpora cavernosa (Andersson and Wagner, 1995). There is a consensus that nitric oxide (NO) is a prerequisite for the generation and maintenance of intracavernous pressure and penile erection (Toda et al., 2005). The formation of NO is accomplished by nitric oxide synthase (NOS) enzyme. The constitutive forms of this enzyme, neuronal NOS (nNOS) and endothelial NOS (eNOS), are involved in the induction of penile erection (Ignarro et al., 1990). $\mathrm{NO}$ is released by nitrergic nerves within the trabecular and arterial tissues as well as by the endothelia of penile arteries (Kim et al., 1991), exerting its relaxing action on corpus cavernosum and penile arteries by activating soluble guanylyl cyclase and increasing intracellular concentration of cGMP (Burnett, 1995; Mizusawa et al., 2002). Subsequently, cGMP-dependent protein kinase I (cGKI or PKG) can alter $\mathrm{Ca}^{2+}$ channel activity and by opening $\mathrm{Ca}^{2+}$-dependent $\mathrm{K}^{+}$channels, lead to hyperpolarization of the smooth muscle cell (Christ et al., 1999). PKG can also phosphorylate other proteins to affect $\mathrm{Ca}^{2+}$ channels or lead to the alteration of the phosphorylation state of myosin light chain, altogether ultimately resulting in NOmediated cavernosal smooth muscle relaxation (Mills et al., 2001). Stimulation of cavernous nerves in anesthetized animals causes penile erection due to the increase of intracavernosal pressure mediated by NO (Burnett et al., 2002; Escrig et al., 1999).

An imbalance between contracting and relaxant factors in the corpus cavernosum, especially through an impairment of the NO system leads to erectile dysfunction (ED), defined as the inability to attain and/or maintain penile erection (NIH Consensus Conference, 1993). ED becomes increasingly prevalent with age, affecting $19-64 \%$ of men aged 40-80 years both in developing and in industrialized countries (for a review see Leite et al., 2007). Other risk factors for ED include the presence of chronic illness (e.g., heart disease, hypertension, diabetes mellitus and depression), smoking, stress, alcohol and drug abuse, obesity and sedentary lifestyle (Leite et al., 2007; Lewis, 2004; Muller and Mulhall, 2006; Priviero et al., 2007).

The current treatment in many cases of ED involves the use of drugs that potentiate compo- nents of the relaxation response. This is the basis for therapy with inhibitors of phosphodiesterase- 5 such as sildenafil, tadalafil and vardenafil that increase cyclic GMP availability, therefore potentiating the NO/cGMP pathway (Leite et al., 2007; Priviero et al., 2007).

The "armed" spider Phoneutria nigriventer is responsible for severe human accidents (Bucaretchi et al., 2000; Vital Brazil et al., 1987) characterized by various symptoms, including neurotoxicity, intense pain, sudoresis, agitation, salivation, priapism, cardiac perturbations characterized by tachycardia, arrhythmia and death (Cordeiro et al., 1992; Schenberg and Pereira Lima, 1966; Vital Brazil et al., 1987). Studies using this venom reported priapism in mice and dogs (Bucaretchi et al., 2000). The venom of this spider is a cocktail of toxins, having peptides, free amino acids, histamine and serotonin (for a review see Cordeiro et al., 1995; Gomez et al., 2002). The partial proteome of this venom was recently described (Richardson et al., 2006). Most of the toxins that have been purified from this venom seem to act on ionic channels, including those for sodium (Araujo et al., 1993; De Lima et al., 2007; Martin-Moutot et al., 2006; Matavel et al., 2002), calcium (Dos Santos et al., 2002; Guatimosim et al., 1997; Leão et al., 2000; Vieira et al., 2005) and potassium (Kushmerick et al., 1999). Some of these toxins have insecticidal activity (for a review see De Lima et al., 2007), interfer with the uptake of L-glutamate on synaptosomes of rat brain (Mafra et al., 1999) and inhibit NMDA-evoked currents in rat hippocampal neurons (Figueiredo et al., 2001). A semi-purified toxic fraction called $\mathrm{PhTx} 2$ releases acetylcholine from rat cerebrocortical synaptosomes (Moura et al., 1998).

It has been shown that some toxins that act on sodium channels, including scorpion toxins, are able to induce relaxation of corpus cavernosum (Teixeira et al., 2003, 2004). The crude venom from $P$. nigriventer induced cavernosal relaxation that could be prevented by L-NAME (Antunes et al., 1993). The PhTx2 fraction contains toxins such as Tx2-5 and Tx2-6 that present a similar structure (Cordeiro et al., 1992) differing in seven amino acid residues. Tx2-5 and Tx2-6 were both described as site 3 toxins, according to their effects on sodium channels (Araujo et al., 1993; Matavel et al., 2002). Male mice injected with $\mathrm{T} \times 2-5$ showed priapism, hypersalivation and high mortality. Pre-treatment with a non-selective NOS inhibitor 
(L-NAME) partially prevented these symptoms, whereas an nNOS-selective inhibitor (7-NI) completely abolished priapism and other effects of Tx25 , including pulmonary edema and death (Yonamine et al., 2004). Tx2-6 toxin when purified and assayed in toxicity tests in mice caused penile erection (Cordeiro and Richardson, 1997, personal communication). In preliminary experiments done in our laboratory we verified that Tx2-6 also causes erection in rats.

In this study we evaluated the effect of the purified toxin Tx2-6 on penile erection of normotensive and mineralocorticoid (deoxycorticosteroneacetate (DOCA)-salt) hypertensive rats. It is well known that the DOCA-salt hypertensive rats present severe erectile dysfunction (Chitaley et al., 2001a, b). We also investigated the effect of Tx2-6 to induce NO release in rat cavernosum tissue using in vivo and confocal microscopy techniques.

\section{Materials and methods}

\subsection{Animals}

All experimental protocols were approved by the Ethics Committee of the Federal University of Minas Gerais (Process no. CETEA 22/2006). Male Wistar rats were provided by CEBIO (Centro de Bioterismo, ICB, Universidade Federal de Minas Gerais), maintained with free access to standard food and drinking water, and kept on a $12 \mathrm{~h}$ light/ dark cycle. Rats (130-170 g) were made hypertensive by uninephrectomy and subcutaneous implantation of a siliastic pellet containing DOCA $(200 \mathrm{mg} / \mathrm{kg}$ of body weight) under tribromoethanol anesthesia. DOCA-salt rats (Chitaley et al., $2001 \mathrm{a}, \mathrm{b})$ received salt water $(1 \% \mathrm{NaCl}, 0.2 \%$ $\mathrm{KCl}$ ) for 4 weeks. Sham-operated rats (uniphrectomized) were used as controls for the DOCA group, and intact age-matched rats were used as regular normotensive rats, both with free access to normal water. Systolic blood pressure was measured by the tail cuff method (pneumatic transducer) after 4 weeks of treatment.

\subsection{Toxin purification}

The toxin Tx2-6 was purified from the venom of the South American 'armed' spider P. nigriventer (Keys) by gel filtration and reverse phase FPLC and HPLC as described by Cordeiro et al. (1992).

\subsection{In vivo measurements of ICP/MAP}

Rats were anesthetized with urethane $(1400 \mathrm{mg} /$ $\mathrm{kg}$; i.p.) and placed on a heating pad. The left femoral artery was exposed and cannulated (using a $30 \mathrm{G}$ needle connected to PE-10 tubing filled with heparinized saline) for continuous monitoring of mean arterial pressure (MAP). The shaft of the penis was freed of skin and fascia, and the right corpus cavernosum was cannulated by insertion of a $30 \mathrm{G}$ needle connected to a pressure transducer, permitting continuous monitoring of corpus cavernosum pressure (ICP) as described elsewhere (Mills et al., 1998). The rat left corpus cavernosum was also cannulated in some animals for administration L-NAME (L- $N{ }^{\mathrm{G}}$-nitro-arginine methyl ester, Sigma) or DAF-FM (diacetate, 4-amino-5-methylamino$2^{\prime}, 7^{\prime}$-difluoroflurescein diacetate, Invitrogen). The abdominal cavity was opened, the right major pelvic ganglion (MPG) exposed, and silver bipolar electrodes were positioned on it for electrical stimulation. Voltage-response curves were performed with continuous stimulation of the MPG (30s duration for each voltage) using a crescent range $(0.5,0.75$, $1.0,1.2,1.5,2.0,2.5$ and $3.0 \mathrm{~V}$ ), a pulse duration of $5 \mathrm{~ms}$ and a frequency of $12 \mathrm{~Hz}$.

The effect of $\mathrm{Tx} 2-6(12 \mu \mathrm{g} / \mathrm{kg}$ s.c. or i.v.) on ganglionic stimulation-induced elevation of $\mathrm{ICP} /$ MAP was evaluated in normotensive control rats. Based on preliminary tests with a range of doses $(3-48 \mu \mathrm{g} / \mathrm{kg})$ the best effect was verified by using $12 \mu \mathrm{g} / \mathrm{kg}$. So, all the experiments in vivo were performed using this dose. In the studies using sham-operated and DOCA-salt hypertensive rats Tx2-6 was administered only subcutaneously. We also investigated the effect of subcutaneous injection of the toxin on ICP/MAP elevation induced by a submaximal stimulation $(1.5 \mathrm{~V})$ in normotensive control rats that received intracavernosal injection of the non-selective NOS inhibitor, L-NAME $(200 \mathrm{mg} / \mathrm{kg})$. The stimulation of MPG was initiated $15 \mathrm{~min}$ after injection of the toxin.

\subsection{Confocal microscopy}

Rats were killed under urethane anesthesia $(1400 \mathrm{mg} / \mathrm{kg}$, i.p.) and corpus cavernosum of normotensive rats was perfused with $10 \mathrm{ml}$ saline (via abdominal aorta) and the penis was rapidly removed. Segments of the corpus cavernosum (approximately $8-10 \mathrm{~mm}$ ) were incubated with Tx2-6 toxin $(0.01 \mu \mathrm{g} / \mathrm{ml})$ and $2.5 \mu \mathrm{M}$ DAF for 
10 min. The segments were washed 3 times with PBS (Physiological buffer solution) every $5 \mathrm{~min}$, and stored at $-80^{\circ} \mathrm{C}$ for $24 \mathrm{~h}$. The frozen penile segments were then embedded with OCT (Tissue-Tek) and sectioned $(20 \mu \mathrm{m}, 6$ slices per animal) with the cryomicrotome at $-20^{\circ} \mathrm{C}$. The slices were fixed at room temperature on gelatinized lamina covered by glycerol $90 \%$ and Tris- $\mathrm{HCl}$ buffer $10 \%$ and maintained frozen until analyzed. Fluorescent images were obtained using a laser scanning confocal microscope (Zeiss 510META). The interaction of DAF-FM with NO released by cells produces green fluorescence under excitation at $488 \mathrm{~nm}$ with argon-ion-laser (oil-immersion objective lens $63 \times$ ). At least one image was captured from each slice. Quantification was performed using National Institutes of Health Image software, version 1.37. Three images were randomly selected and submitted to quantification analysis. The RGB confocal images were loaded into the program and converted to 8-bit gray scale before subtracting background fluorescence equivalently for all images (setting the threshold to $50 \%$ maximum intensity). Three regions of the captured images were then outlined on the gray-scale pictures, and the fluorescence density was measured. Values are expressed as the mean of fluorescence intensity per square micrometer of the respective images.

In another protocol a normotensive rat received Tx2-6 (48 $\mu \mathrm{g} / \mathrm{kg}$, i.v.), and after $10 \mathrm{~min}$ the penis was infused $(1 \mu \mathrm{l} / \mathrm{min})$ with $10 \mu \mathrm{l}$ DAF-FM $(2.5 \mu \mathrm{M})$. After euthanasia the rat penis was perfused with $10 \mathrm{ml}$ saline (via abdominal aorta), rapidly removed and cleaned from connective tissue. A segment of $10 \mathrm{~mm}$ of the cavernosum tissue was taken from the penis, stored at $-80{ }^{\circ} \mathrm{C}$ for $24 \mathrm{~h}$ and processed as described above. In another set of experiments penile segments were obtained and processed, as described above, from sham-operated and DOCAsalt hypertensive rats after a full voltage-response curve during DAF-FM infusion, and after $10 \mathrm{~min}$ exposure to Tx2-6 (12 $\mu \mathrm{g} / \mathrm{kg}$, s.c.) followed by a full voltage-response curve during DAF-FM infusion.

\subsection{Statistical analyses}

Results were expressed as mean \pm SEM. Statistical analyses were performed using two-way analyses of variance (ANOVA) followed by Bonferroni post hoc test for multiple comparisons. Systolic blood pressure and fluorescence intensity data were analyzed by Student's $t$ - test for unpaired compar- ison. A value of $p<0.05$ was considered statistically significant.

\section{Results}

\subsection{Tx2-6 potentiates rat erectile function after subcutaneous or intravenous injections}

To examine the effect of Tx2-6 on penile erectile function of normotensive control rats, voltageresponse curves $(0.5-3.0 \mathrm{~V}, 12 \mathrm{~Hz}, 0.1 \mathrm{~ms}, 30 \mathrm{~s}$ each step) were performed before and $15 \mathrm{~min}$ after injection of the toxin $(12 \mu \mathrm{g} / \mathrm{kg}$, s.c. or i.v.). The erectile response, represented as the ICP/MAP ratio, was significantly potentiated after subcutaneous (Fig. 1A) or intravenous injection of Tx2-6 (Fig. 1B).

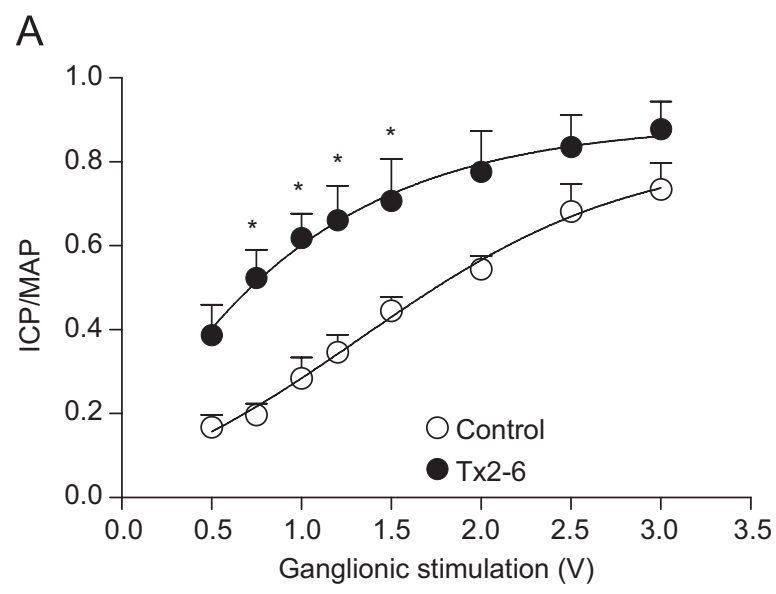

B

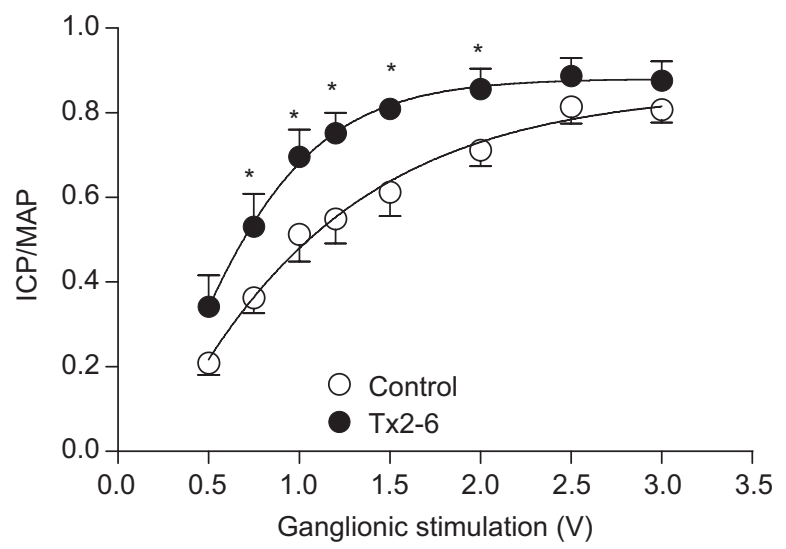

Fig. 1. Rat penile erection induced by ganglionic stimulation in normotensive control rats after subcutaneous or intravenous injection of Tx2-6. Ganglionic stimulation $(0.5-3.0 \mathrm{~V})$ induces an increase in ICP/MAP ratio which was significantly potentiated by (panel A) subcutaneous $(n=6)$ or (panel B) intravenous $(n=5)$ injection of Tx2-6 (12 $\mu \mathrm{g} / \mathrm{kg}) . \quad * P<0.05$ (two-way ANOVA followed by Bonferroni test). 
Injection of $\mathrm{Tx} 2-6$ in absence of ganglionic stimulation also increased the ICP/MAP ratio (not shown).

\subsection{Tx2-6-induced potentiation of erectile function depends on $\mathrm{NO}$ release}

To determine whether the potentiating effect of Tx2-6 on erectile function was NO-dependent, we performed additional experiments in the presence of the NOS inhibitor, L-NAME. Fig. 2A shows that subcutaneous injection of Tx2-6 $(12 \mu \mathrm{g} / \mathrm{kg})$ significantly increased the ICP/MAP ratio induced by submaximal electrical stimulation $(1.5 \mathrm{~V})$ of the MPG. Administration of L-NAME, at a dose of

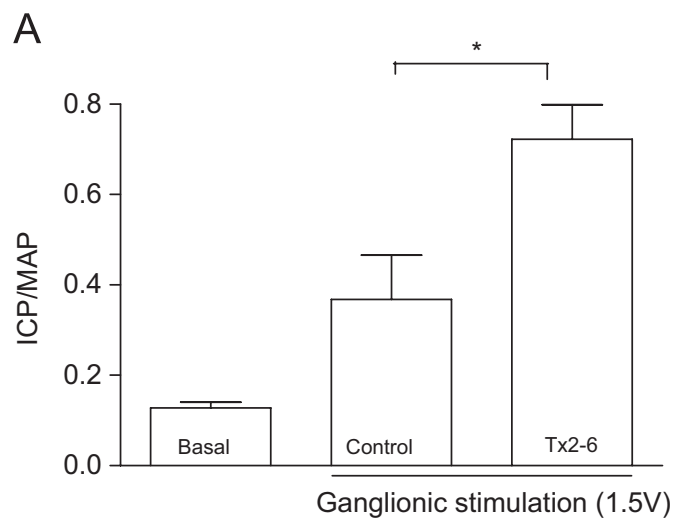

B

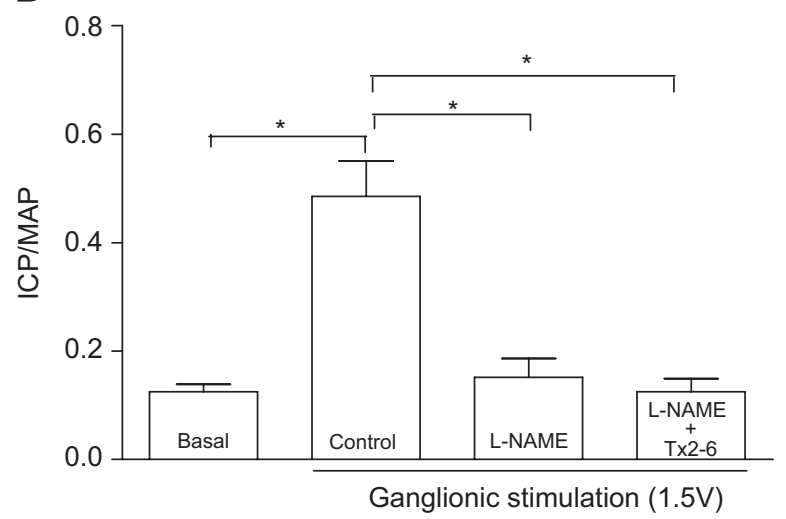

Fig. 2. Inhibition of rat erectile function by L-NAME, a nonselective NOS inhibitor, is not restored by Tx2-6. (Panel A) ICP MAP ratio elevation (control) induced by submaximal $(1.5 \mathrm{~V})$ ganglionic stimulation is significantly potentiated by subcutaneous Tx2-6. (Panel B) Treatment with L-NAME $(200 \mathrm{mg} / \mathrm{kg}$, intracavernosal) caused a significant decrease in ICP/MAP ratio elevation induced by submaximal stimulation. This effect was not overcome by the subcutaneous injection of Tx2-6 $(12 \mu \mathrm{g} / \mathrm{kg})$. ${ }^{*} P<0.05$ (Two-way ANOVA followed by Bonferroni test).
$200 \mu \mathrm{g} / \mathrm{kg}$, into the left cavernosal sinuses did not alter baseline ICP or MAP (data not shown). However, as previously reported (Chitaley et al., 2001b), L-NAME injection significantly attenuated the increase in ICP/MAP induced by a submaximal stimulus (ICP/MAP: Control, $0.48 \pm 0.07$ vs L-NAME, $0.15 \pm 0.03 ; P<0.05, n=6$ ), which lasted for at least $30 \mathrm{~min}$ after administration. More importantly, the attenuated erectile response induced by NOS inhibition was not altered by administration of Tx2-6, suggesting that NO is an important downstream mediator of the Tx2-6 potentiating effect in this experimental model (Fig. 2B).

\subsection{Tx2-6 induces and facilitates $N O$ release in rat corpus cavernosum}

To confirm the possibility that $\mathrm{T} \times 2-6$ causes release of NO in the corpus cavernosum, the NO indicator DAF-FM was used. NO release in slices of corpus cavernosum from normotensive control rats was increased after $10 \mathrm{~min}$ incubation with 0.01 $\mu \mathrm{g} / \mathrm{ml}$ Tx2-6 as observed in the representative figures (Fig. 3A), a result corroborated by the quantification of the florescence intensity (Fig. 3B). Intravenous injection of Tx2-6 $(48 \mu \mathrm{g} / \mathrm{kg})$ induced substantial release of $\mathrm{NO}$ in the sinusoids of cavernosum tissue from normotensive control animals as observed in the representative figure (Fig. 3C) and corroborated by the quantification of the florescence intensity (Fig. 3D). MPG stimulation or subcutaneous injection of Tx2-6 $(12 \mu \mathrm{g} / \mathrm{kg})$ alone induced NO release in cavernosum tissue (data not shown). Moreover, a combination of ganglionic stimulation and Tx2-6 evoked a more pronounced release of $\mathrm{NO}$ in the penile tissue from sham-operated and DOCA-salt hypertensive rats (Fig. 5).

\subsection{Tx2-6 reverses erectile dysfunction in DOCA- salt hypertensive rats}

To test whether Tx2-6 could ameliorate ED, we determined its effect in DOCA-salt hypertensive rats (systolic blood pressure: $194 \pm 3 \mathrm{mmHg}$ vs $100 \pm 4 \mathrm{mmHg} ; P<0.05$, DOCA vs sham, $n=8$ and 9 , respectively), which presented severe ED compared with sham-operated rats (Fig. 4A and B), in accordance with previous data from our laboratory (Chitaley et al., 2001a). Subcutaneous administration of $\mathrm{Tx} 2-6 \quad(12 \mu \mathrm{g} / \mathrm{kg})$ increased the electrically induced elevation of ICP/MAP in both 
A
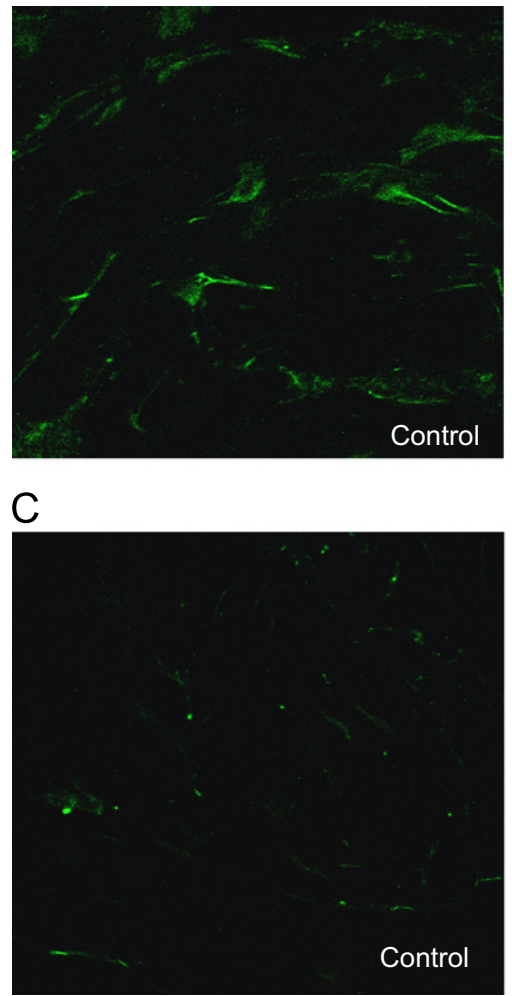
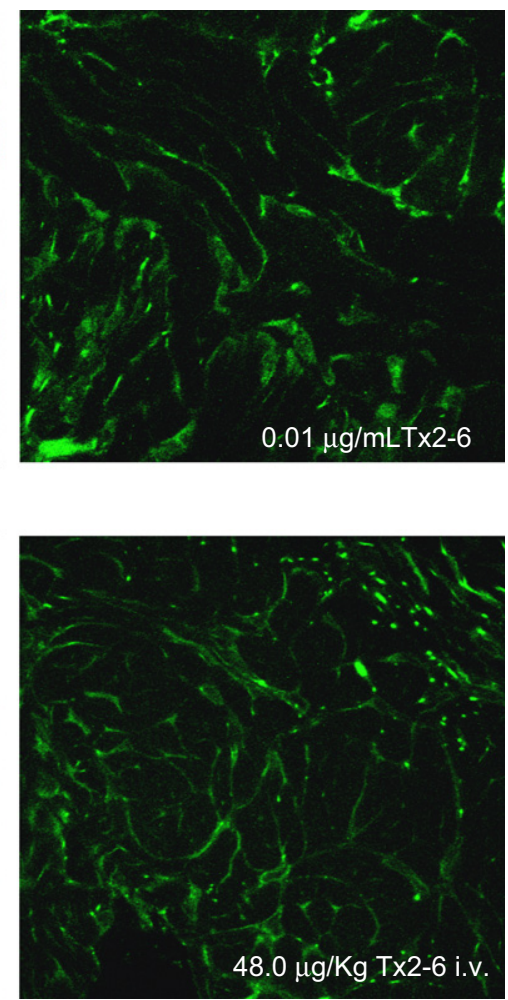

B

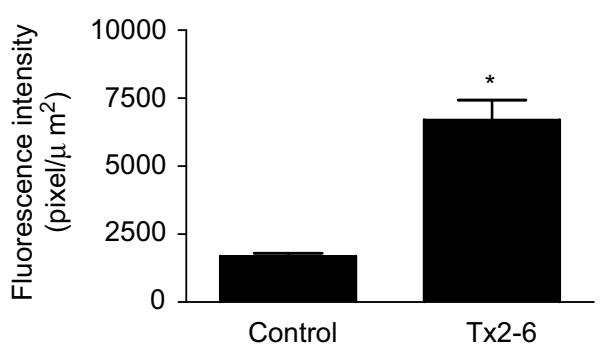

D

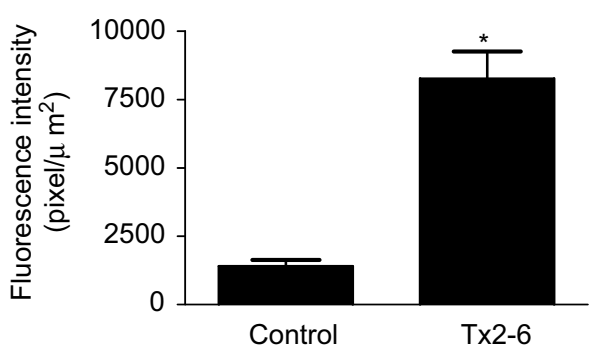

Fig. 3. Increase in NO release in corpus cavernosum from normotensive control rats induced by Tx2-6 observed using the DAF-FM technique. (Panel A) In vitro incubation with Tx2-6 $(0.01 \mu \mathrm{g} / \mathrm{ml})$ induced $\mathrm{NO}$ release in slice of cavernosum tissue. (Panel B) Increased fluorescence intensity observed in rat corpus cavernosum slices incubated with Tx2-6. (Panel C) Intravenous injection of Tx2-6 (48 $\mu \mathrm{g} / \mathrm{kg}$ ) caused NO release in corpus cavernosum. (Panel D) Increased fluorescence intensity observed in rat corpus cavernosum from normotensive control rats intravenously injected with Tx2-6. Results are expressed as means $\pm \mathrm{SEM} ; n=3 .{ }^{*} P<0.05$ (Student's $t$ - test).

sham-operated (Fig. 4A) and DOCA-salt hypertensive rats (Fig. 4B). Furthermore, Tx2-6 treatment completely normalized the erectile function of DOCA-salt rats (Fig. 4B).

\section{Discussion}

The venom of the spider $P$. nigriventer has a cocktail of toxins active on mammal cells, including several neurotoxins that exert diverse biological effects. Human response to this spider venom is characterized by various symptoms, one of which is priapism.

This is the first study to demonstrate the effect of the toxin Tx2-6 purified from the venom of the spider $P$. nigriventer on rat erectile function. In the present study, we examined the effect of Tx2-6 on rat erectile function using an in vivo model, the effect of this toxin on the release of $\mathrm{NO}$ in rat corpus cavernosum and the potentiation effect of
Tx2-6 on penile erection of animals presenting severe ED such as the DOCA-salt hypertensive rats.

The potentiating effect of $T \times 2-6$ on rat penile erection seems to be mediated by relaxation of the vasculature and smooth muscle in the corpus cavernosum induced by the release of NO. This important relaxant agent could be synthesized in nerve terminals and endothelial cells lining blood vessels and lacuna spaces in the corpus cavernosum (Burnett et al., 1992). The crucial role of NO derived from non-andrenergic, non-cholinergic (NANC) nerves and possibly from the corpus cavernosum sinusoidal endothelium in penile erection is widely accepted (Toda et al., 2005). The mechanisms involved in the regulation of either contraction or relaxation of the corpus cavernosum and penile vasculature have been intensely investigated during the past decade (Andersson and Wagner, 1995). NO has been suggested as the main mediator of NANC nerve-induced relaxation of the corpora cavernosa 
A

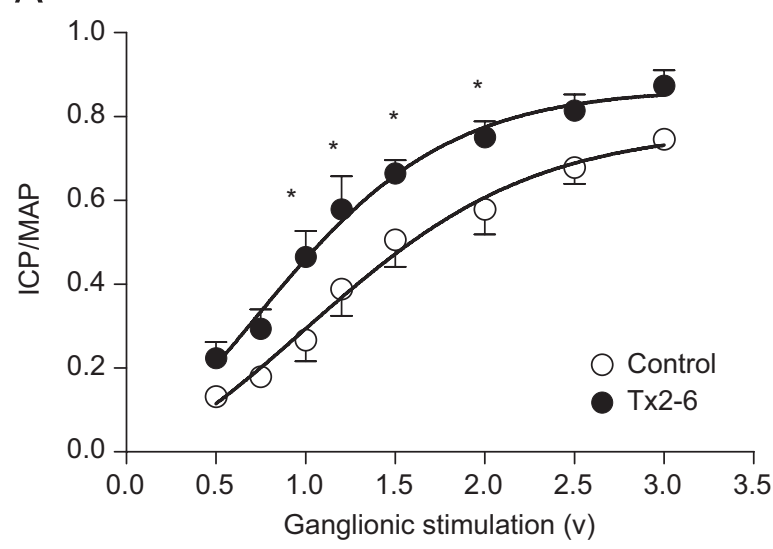

B

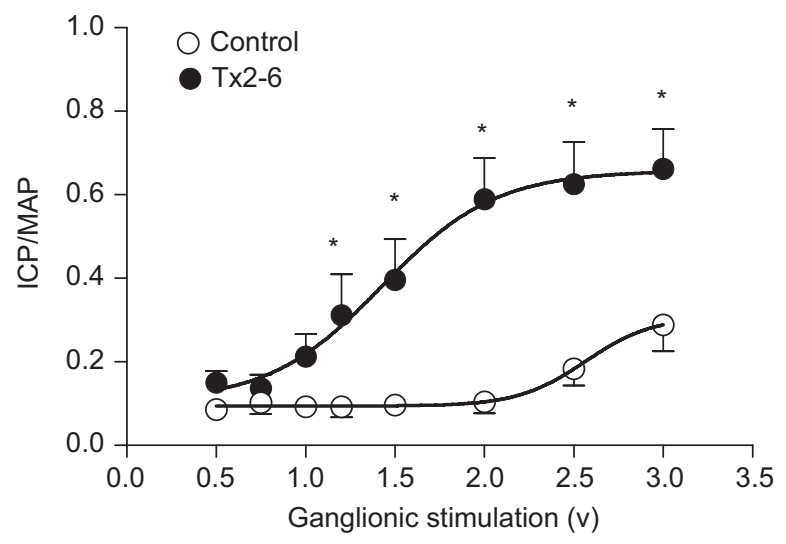

Fig. 4. Penile erection induced by ganglionic stimulation in DOCA-salt hypertensive rats after subcutaneous injection of Tx2-6. (Panel A). Ganglionic stimulation (0.5-3.0 V) induces an increase in ICP/MAP in sham-operated rats that was facilitated by subcutaneous injection of Tx2-6 (12 $\mu \mathrm{g} / \mathrm{kg}, n=9)$. (Panel B). DOCA-salt hypertensive rats exhibited severe impairment on ICP/MAP upon stimulation of the MPG that was completely restored to sham-operated levels after subcutaneous injection of Tx2-6 $(12 \mu \mathrm{g} / \mathrm{kg}, n=6)$. ${ }^{*} P<0.05$ (Two-way ANOVA followed by Bonferroni test).

in vitro, as well as of increased intracavernous pressure in vivo in a variety of mammals, including rats (Burnett et al., 1992), rabbits (Teixeira et al., 1998), dogs (Hayashida et al., 1996), monkeys (Okamura et al., 1998) and humans (Leone et al., 1994).

$P$. nigriventer venom causes activation of voltagedependent sodium channels in muscle and nerve cell membranes (Cordeiro et al., 1995; Gomez et al., 2002). Teixeira et al. (2003) showed that this spider venom is active on vascular smooth muscle mainly due to increased influx of calcium from extracellular sources. The PhTx2-6 fraction has been proposed to be the main toxic fraction of the $P$. nigriventer spider venom, responsible for increasing permeability of sodium channels (Mattiello-Sverzuta and Cruz-Hofling, 2000).

It has been demonstrated that both the fraction PhTx2-6 and the purified toxin (Tx2-6) act on sodium channels slowing down its inactivation (Araujo et al., 1993; Matavel et al., 2002), an effect similar to that observed for $\alpha$-toxins from arthropods (Cestele and Catterall, 2000). This change in the inactivation time would maintain a depolarized state of the nerve fibers resulting in an increase in calcium influx and consequently an increase in neurotransmitters release, as observed for acetylcholine (Moura et al., 1998). Action potential opens tetrodotoxin-sensitive sodium channels in nitrergic nerve terminals and promote the $\mathrm{Ca}^{2+}$ influx possibly through $\mathrm{N}$-type $\mathrm{Ca}^{2+}$ channels in blood vessels (Toda et al., 2005). This seems to be the case in the corpus cavernosum, because relaxations elicited by nerve stimulation of human and canine corpus cavernosum strips were sensitive to $\omega$-conotoxin, an $\mathrm{N}$-type $\mathrm{Ca}^{2+}$ channel blocker (Leone et al., 1994; Okamura et al., 2001). Increase in cytosolic calcium participates in activation of nNOS in the presence of calmodulin (Bredt and Snyder, 1990). Neuronal release or synthesis of NO is dependent on intracellular bioavailability of $\mathrm{Ca}^{2+}$ in the rabbit corpus cavernosum (Saito et al., 1996).

As mentioned before, intra-peritoneal injections of Tx2-5, a toxin that differs of Tx2-6 by seven amino acid residues, induced a toxic syndrome that included priapism and death, these effects being partially prevented by L-NAME and abolished by the nNOS-selective inhibitor 7-NI (Yonamine et al., 2004). Studies of structure-function are of fundamental importance to clarify the activities of these toxins, concerning toxicity and potentiating of erection. Furthermore, possible differences within species, i.e. mice and rats, would be evaluated. Considering these data one could speculate that the potentiated erection induced by Tx2-6 could also point out to an important role of nNOS on the increased $\mathrm{NO}$ release. Further experiments are needed to confirm this conclusion.

There has been evidence that penile erection is driven by two distinct hemodynamic stages. The first one is dependent on NO release from NANC nerves in the penis causing dilation of arterioles and expansion of sinusoids. This stage is followed by the 


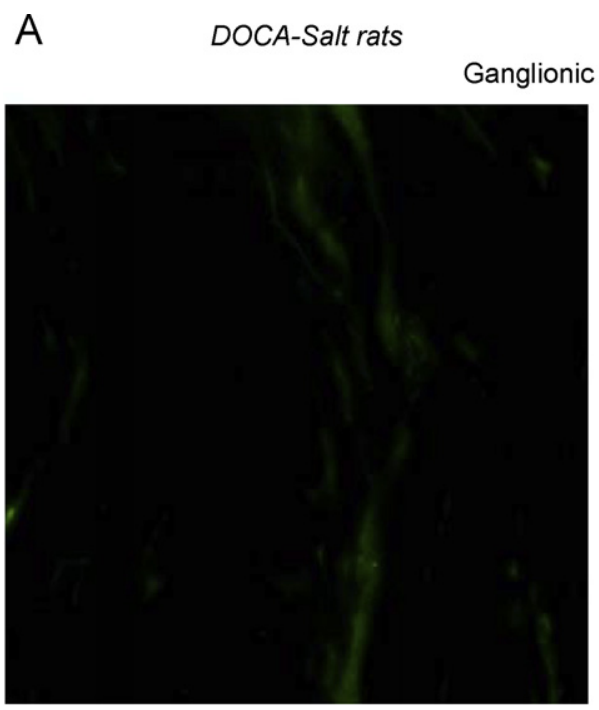

B

C

D
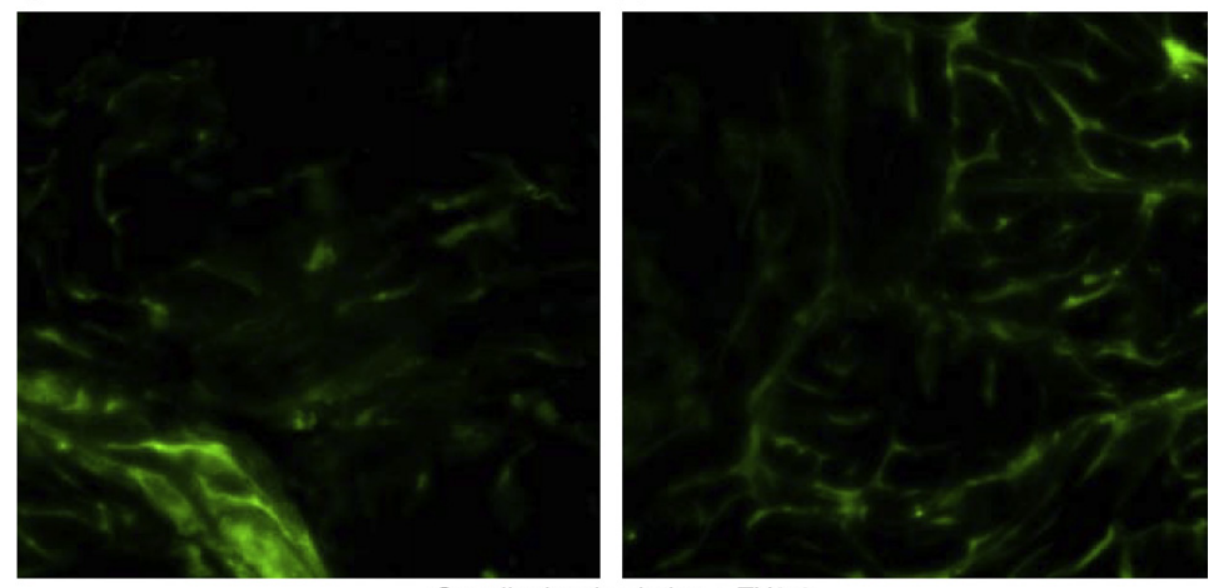

Ganglionic stimulation + TX2-6

Fig. 5. Increase in NO release induced by ganglionic stimulation (Panels A and B) or ganglionic stimulation plus Tx2-6 (Panels C and D) in corpus cavernosum from sham-operated rats (Panels B and D) and DOCA-salt hypertensive rats (Panels A and C). Subcutaneous injection of Tx2-6 $(12 \mu \mathrm{g} / \mathrm{kg})$ potentiates the increase in NO release induced by ganglionic stimulation in cavernosum tissue from shamoperated and DOCA-salt hypertensive rats. DAF-FM technique was used as described.

release of endothelium-derived relaxing factor from endothelial cells surrounding corpus cavernosum trabecules in response to shear stress. It is generally accepted that NO derived from eNOS is the major vasodilator for this second stage of erection (Burnett et al., 1992; Hurt et al., 2002). From our results, it is not clear yet whether Tx2-6 acts to stimulate NO release from NANC nerve terminals, to facilitate the release of acetylcholine from preand post-synaptic parasympathetic nerves, or to sensitize the corpus cavernosum endothelial cells to the increase of shear stress. Additional studies are required to reveal the precise mechanism by which Tx2-6 induces NO release facilitates penile erection.
The erection induced by Tx2-6 could be observed about 15 min after subcutaneous injection. It can be the necessary time for the toxin to reach its target. Preliminary experiments using Tx2-6 labeled with technetium-99 (data not shown) revealed that after $10 \mathrm{~min}$ the distribution of labeled toxin is predominantly in kidney and bladder. It is worth mentioning that this toxin was also found in the penile tissue. This suggests a possible existence of specific receptors on corpus cavernosum for the toxin. Other experiments are being carried out to clarify this point.

Our results clearly demonstrated for the firs time, that $\mathrm{Tx} 2-6$, a toxin purified from the venom of the 
$P$. nigriventer spider can induce erection and facilitate the ganglionic-stimulated penile erection in control rats. We have also shown that this toxin is able to induce and facilitate the release of NO from cavernosum tissue of normotensive and hypertensive rats. Moreover, the severely depressed erectile function of DOCA-salt hypertensive rats was essentially normalized by Tx2-6 administration. These data suggest that $\mathrm{T} \times 2-6$ is a potential tool for the development of new therapeutics for the treatment of ED.

\section{Acknowledgments}

This work was supported in part by Conselho Nacional de Desenvolvimento Científico e Tecnológico (CNPq) and Fundação de Amparo à Pesquisa do Estado de Minas Gerais (FAPEMIG). K.P. Nunes and L.F. Lanza are recipients of research scholarships from CNPq. M.E. De Lima and A.M.C. Pimenta are recipients of fellowships from CNPq. R. Leite and R.C. Webb are supported by NIH grant (HL71138).

\section{References}

Andersson, K.E., Wagner, G., 1995. Physiology of penile erection. Physiol. Rev. 75, 191.

Antunes, E., Marangoni, R.A., Giglio, J.R., Brain, S.D., de Nucci, G., 1993. Activation of tissue kallikrein-kininogenkinin system in rabbit skin by a fraction isolated from Phoneutria nigriventer (armed spider) venom. Toxicon 31, 1385.

Araujo, D.A., Cordeiro, M.N., Diniz, C.R., Beirao, P.S., 1993. Effects of a toxic fraction, PhTx2, from the spider Phoneutria nigriventer on the sodium current. Naunyn Schmiedebergs Arch. Pharmacol. 347, 205.

Bredt, D.S., Snyder, S.H., 1990. Isolation of nitric oxide synthetase, a calmodulin-requiring enzyme. Proc. Natl. Acad. Sci. USA 87, 682.

Bucaretchi, F., Deus Reinaldo, C.R., Hyslop, S., Madureira, P.R., De Capitani, E.M., Vieira, R.J., 2000. A clinicoepidemiological study of bites by spiders of the genus Phoneutria. Rev. Inst. Med. Trop. Sao Paulo 42, 17.

Burnett, A.L., 1995. Role of nitric oxide in the physiology of erection. Biol. Reprod. 52, 485.

Burnett, A.L., Lowenstein, C.J., Bredt, D.S., Chang, T.S., Snyder, S.H., 1992. Nitric oxide: a physiologic mediator of penile erection. Science 257, 401.

Burnett, A.L., Chang, A.G., Crone, J.K., Huang, P.L., Sezen, S.E., 2002. Noncholinergic penile erection in mice lacking the gene for endothelial nitric oxide synthase. J. Androl. 23, 92.

Cestele, S., Catterall, W.A., 2000. Molecular mechanisms of neurotoxin action on voltage-gated sodium channels. Biochimie 82,883 .
Chitaley, K., Webb, R.C., Dorrance, A.M., Mills, T.M., $2001 \mathrm{a}$. Decreased penile erection in DOCA-salt and stroke pronespontaneously hypertensive rats. Int. J. Impot. Res. 13 (Suppl. 5), S16.

Chitaley, K., Wingard, C.J., Clinton Webb, R., Branam, H., Stopper, V.S., Lewis, R.W., Mills, T.M., 2001b. Antagonism of rho-kinase stimulates rat penile erection via a nitric oxideindependent pathway. Nat. Med. 7, 119.

Christ, G.J., Wang, H.Z., Venkateswarlu, K., Zhao, W., Day, N.S., 1999. Ion channels and gap junctions: their role in erectile physiology, dysfunction, and future therapy. Mol. Urol. 3, 61 .

Cordeiro, M.N., Diniz, C.R., Valentim Ado, C., von Eickstedt, V.R., Gilroy, J., Richardson, M., 1992. The purification and amino acid sequences of four Tx2 neurotoxins from the venom of the Brazilian 'armed' spider Phoneutria nigriventer (Keys). FEBS Lett. 310, 153.

Cordeiro, M.N., Richardson, M., Gilroy, J., Figueiredo, S.G.D., Beirão, P.S.L., Diniz, C.R., 1995. Properties of the venom from the South American armed spider Phoneutria nigriventer (Keyserling, 1891). J. Toxicol. Toxin Rev. 14, 309.

De Lima, M.E., Figueiredo, S.G., Pimenta, A.M., Santos, D.M., Borges, M.H., Cordeiro, M.N., Richardson, M., Oliveira, L.C., Stankiewicz, M., Pelhate, M., 2007. Peptides of arachnid venoms with insecticidal activity targeting sodium channels. Comp. Biochem. Physiol. C Toxicol. Pharmacol.

Dos Santos, R.G., Van Renterghem, C., Martin-Moutot, N., Mansuelle, P., Cordeiro, M.N., Diniz, C.R., Mori, Y., De Lima, M.E., Seagar, M., 2002. Phoneutria nigriventer omegaphonetoxin IIA blocks the Cav2 family of calcium channels and interacts with omega-conotoxin-binding sites. J. Biol. Chem. 277, 13856.

Escrig, A., Gonzalez-Mora, J.L., Mas, M., 1999. Nitric oxide release in penile corpora cavernosa in a rat model of erection. J. Physiol. 516 (Pt. 1), 261.

Figueiredo, S.G., de Lima, M.E., Cordeiro, NascimentoM., Diniz, C.R., Patten, D., Halliwell, R.F., Gilroy, J., Richardson, M., 2001. Purification and amino acid sequence of a highly insecticidal toxin from the venom of the Brazilian spider Phoneutria nigriventer which inhibits NMDA-evoked currents in rat hippocampal neurons. Toxicon 39, 309.

Gomez, M.V., Kalapothakis, E., Guatimosim, C., Prado, M.A., 2002. Phoneutria nigriventer venom: a cocktail of toxins that affect ion channels. Cell Mol. Neurobiol. 22, 579.

Guatimosim, C., Romano-Silva, M.A., Cruz, J.S., Beirao, P.S., Kalapothakis, E., Moraes-Santos, T., Cordeiro, M.N., Diniz, C.R., Gomez, M.V., Prado, M.A., 1997. A toxin from the spider Phoneutria nigriventer that blocks calcium channels coupled to exocytosis. Br. J. Pharmacol. 122, 591.

Hayashida, H., Okamura, T., Tomoyoshi, T., Toda, N., 1996. Neurogenic nitric oxide mediates relaxation of canine corpus cavernosum. J. Urol. 155, 1122.

Hurt, K.J., Musicki, B., Palese, M.A., Crone, J.K., Becker, R.E., Moriarity, J.L., Snyder, S.H., Burnett, A.L., 2002. Aktdependent phosphorylation of endothelial nitric-oxide synthase mediates penile erection. Proc. Natl. Acad. Sci. USA 99, 4061.

Ignarro, L.J., Bush, P.A., Buga, G.M., Wood, K.S., Fukuto, J.M., Rajfer, J., 1990. Nitric oxide and cyclic GMP formation upon electrical field stimulation cause relaxation of corpus cavernosum smooth muscle. Biochem. Biophys. Res. Commun. 170,843 . 
Kim, N., Azadzoi, K.M., Goldstein, I., Saenz de Tejada, I., 1991. A nitric oxide-like factor mediates nonadrenergic-noncholinergic neurogenic relaxation of penile corpus cavernosum smooth muscle. J. Clin. Invest. 88, 112.

Kushmerick, C., Kalapothakis, E., Beirao, P.S., Penaforte, C.L., Prado, V.F., Cruz, J.S., Diniz, C.R., Cordeiro, M.N., Gomez, M.V., Romano-Silva, M.A., Prado, M.A., 1999. Phoneutria nigriventer toxin Tx3-1 blocks A-type $\mathrm{K}^{+}$currents controlling $\mathrm{Ca}^{2+}$ oscillation frequency in GH3 cells. J. Neurochem. 72, 1472.

Leao, R.M., Cruz, J.S., Diniz, C.R., Cordeiro, M.N., Beirao, P.S., 2000. Inhibition of neuronal high-voltage activated calcium channels by the omega-Phoneutria nigriventer Tx3-3 peptide toxin. Neuropharmacology 39, 1756.

Leite, R., Giachini, F.R., Carneiro, F.S., Nunes, K.P., Tostes, R.C., Webb, R.C., 2007. Targets for the treatment of erectile dysfunction: is NO/cGMP still the answer? Recent Patents Cardiovasc. Drug Discov. 2, 119.

Leone, A.M., Wiklund, N.P., Hokfelt, T., Brundin, L., Moncada, S., 1994. Release of nitric oxide by nerve stimulation in the human urogenital tract. Neuroreport 5, 733.

Lewis, R.W., 2004. Definitions, Classification, and Epidemiology of Sexual Dysfunction. Health Publications, Paris, p. 39.

Mafra, R.A., Figueiredo, S.G., Diniz, C.R., Cordeiro, M.N., Cruz, J.D., De Lima, M.E., 1999. PhTx4, a new class of toxins from Phoneutria nigriventer spider venom, inhibits the glutamate uptake in rat brain synaptosomes. Brain Res. 831, 297.

Martin-Moutot, N., Mansuelle, P., Alcaraz, G., Dos Santos, R.G., Cordeiro, M.N., De Lima, M.E., Seagar, M., Van Renterghem, C., 2006. Phoneutria nigriventer toxin 1: a novel, state-dependent inhibitor of neuronal sodium channels that interacts with micro conotoxin binding sites. Mol. Pharmacol. 69, 1931.

Matavel, A., Cruz, J.S., Penaforte, C.L., Araujo, D.A., Kalapothakis, E., Prado, V.F., Diniz, C.R., Cordeiro, M.N., Beirao, P.S., 2002. Electrophysiological characterization and molecular identification of the Phoneutria nigriventer peptide toxin PnTx2-6. FEBS Lett. 523, 219.

Mattiello-Sverzuta, A.C., Cruz-Hofling, M.A., 2000. Toxin 2 (PhTx2), a neurotoxic fraction from Phoneutria nigriventer spider venom, causes acute morphological changes in mouse skeletal muscle. Toxicon 38, 793.

Mills, T.M., Lewis, R.W., Stopper, V.S., 1998. Androgenic maintenance of inflow and veno-occlusion during erection in the rat. Biol. Reprod. 59, 1413.

Mills, T.M., Chitaley, K., Wingard, C.J., Lewis, R.W., Webb, R.C., 2001. Effect of rho-kinase inhibition on vasoconstriction in the penile circulation. J. Appl. Physiol. 91, 1269.

Mizusawa, H., Hedlund, P., Brioni, J.D., Sullivan, J.P., Andersson, K.E., 2002. Nitric oxide independent activation of guanylate cyclase by $\mathrm{YC}-1$ causes erectile responses in the rat. J. Urol. 167, 2276.

Moura, J.R., Prado, M.A., Gomez, M.V., Kalapothakis, E., Diniz, C.R., Cordeiro, M.N., Romano-Silva, M.A., 1998. Investigation of the effect of PhTx2, from the venom of the spider Phoneutria nigriventer, on the release of $[3 \mathrm{H}]$-acetylcholine from rat cerebrocortical synaptosomes. Toxicon $36,1189$.
Muller, A., Mulhall, J.P., 2006. Cardiovascular disease, metabolic syndrome and erectile dysfunction. Curr. Opin. Urol. 16,435 .

NIH Consensus Conference, 1993. Impotence. NIH Consensus Development Panel on Impotence. JAMA 270, 83.

Okamura, T., Ayajiki, K., Toda, N., 1998. Monkey corpus cavernosum relaxation mediated by $\mathrm{NO}$ and other relaxing factor derived from nerves. Am. J. Physiol. 274, H1075.

Okamura, T., Fujioka, H., Ayajiki, K., 2001. Effects of calcium antagonists on the nitrergic nerve function in canine corpus cavernosum. Jpn. J. Pharmacol. 87, 208.

Priviero, F.B., Leite, R., Webb, R.C., Teixeira, C.E., 2007. Neurophysiological basis of penile erection. Acta Pharmacol. Sin. 28, 751.

Richardson, M., Pimenta, A.M., Bemquerer, M.P., Santoro, M.M., Beirao, P.S., Lima, M.E., Figueiredo, S.G., Bloch Jr., C., Vasconcelos, E.A., Campos, F.A., Gomes, P.C., Cordeiro, M.N., 2006. Comparison of the partial proteomes of the venoms of Brazilian spiders of the genus Phoneutria. Comp. Biochem. Physiol. C Toxicol. Pharmacol. 142 (3-4), 173-187.

Saito, M., Broderick, G., Wein, A.J., Levin, R.M., 1996. Effect of alteration in the extracellular potassium and calcium on fieldstimulated relaxation of the rabbit corpus cavernosum. Gen. Pharmacol. 27, 375.

Schenberg, S., Pereira Lima, F.A., 1966. Pharmacology of the polypeptides from the venom of spider Phoneutria fera. Mem. Inst. Butantan 33, 627.

Teixeira, C.E., Bento, A.C., Lopes-Martins, R.A., Teixeira, S.A., von Eickestedt, V., Muscara, M.N., Arantes, E.C., Giglio, J.R., Antunes, E., de Nucci, G., 1998. Effect of Tityus serrulatus scorpion venom on the rabbit isolated corpus cavernosum and the involvement of NANC nitrergic nerve fibres. Br. J. Pharmacol. 123, 435.

Teixeira, C.E., Ifa, D.R., Corso, G., Santagada, V., Caliendo, G., Antunes, E., De Nucci, G., 2003. Sequence and structure-activity relationship of a scorpion venom toxin with nitrergic activity in rabbit corpus cavernosum. FASEB J. 17, 485.

Teixeira, C.E., de Oliveira, J.F., Baracat, J.S., Priviero, F.B., Okuyama, C.E., Rodrigues Netto Jr., N., Fregonesi, A., Antunes, E., De Nucci, G., 2004. Nitric oxide release from human corpus cavernosum induced by a purified scorpion toxin. Urology 63, 184

Toda, N., Ayajiki, K., Okamura, T., 2005. Nitric oxide and penile erectile function. Pharmacol. Ther. 106, 233.

Vieira, L.B., Kushmerick, C., Hildebrand, M.E., Garcia, E., Stea, A., Cordeiro, M.N., Richardson, M., Gomez, M.V., Snutch, T.P., 2005. Inhibition of high voltage-activated calcium channels by spider toxin PnTx3-6. J. Pharmacol. Exp. Ther. 314, 1370 .

Vital Brazil, O., Bernado Leite, G., Fontana, M.D., 1987. Modo de ação da peçonha de armadeira (Phoneutria nigriventer) na aurícula isolada de cobaia. In: Reunião Anual da Federação das Sociedades de Biologica Experimental 2.

Yonamine, C.M., Troncone, L.R., Camillo, M.A., 2004. Blockade of neuronal nitric oxide synthase abolishes the toxic effects of Tx2-5, a lethal Phoneutria nigriventer spider toxin. Toxicon 44, 169. 